\title{
Correlation of Mucin 1 (MUC1) immunohistochemical expression with histopathological grading and molecular subtypes in invasive breast carcinoma
}

\author{
Fajriani, Delyuzar, Betty, Joko S. Lukito, Lidya Imelda Laksmi \\ fajrianidr@gmail.com \\ Department of Anatomic Pathology, Faculty of Medicine, Universitas Sumatera Utara, Medan, Indonesia
}

\begin{abstract}
Background: Breast carcinoma is a malignancy that occurs from breast cells that grow and continue to proliferate uncontrollably. Mucin 1 (MUC1) has become a topic of interest in cancer treatment due to its upregulation, which affects the invasion, proliferation, and survival of tumor cells, by reducing cells adhesion and extracellular cell-matrix adhesion.

Objective: To analyze the correlation between the immunohistochemical expression of Mucin 1 (MUC1) with histopathological grading and molecular subtypes in invasive breast carcinoma.

Methods: Formalin-fixed tissue paraffin blocks from 42 patients with invasive breast carcinoma were used as research samples and Mucin 1 (MUC1) immunohistochemical staining was assessed. The correlation between Mucin 1 (MUC1) expression and histopathological grading and molecular subtypes was analyzed by statistical tests using the SPSS program.

Results: Of the 42 samples studied, for the age group, the most found were in the age group 40-49 years and the age group 50-59 years (each with 35.7\%), while the age group 20-29 years was the least (2.4\%). The most histopathological subtypes were invasive carcinoma of no special type (85.7\%), with grade 2 being the most common (42.8\%). The most common molecular subtype was luminal B (40.5\%). Mucin 1 (MUC1) was expressed in $73.8 \%$ of invasive breast carcinoma specimens.

Conclusion: There is a significant unidirectional correlation between the immunohistochemical expression of Mucin 1 (MUC1), either with histopathological grading or with molecular subtypes in invasive breast carcinoma ( $\mathrm{p}$-value were 0.027 and 0.010 , respectively).
\end{abstract}

Keywords: Mucin 1 (MUC1), immunohistochemical, invasive breast carcinoma, histopathological grading, molecular subtypes

\section{Introduction}

Breast cancer is the most commonly diagnosed cancer in women globally, and it has remained an important health challenge for decades, with an estimated 2 million new cases diagnosed and more than half a million deaths. This shows that breast cancer has proven to be a major barrier to increasing life expectancy worldwide [1]. Breast cancer itself is a malignancy that occurs from breast epithelial cells that grow and continue to proliferate uncontrollably [2].

Based on data from the Global Burden of Cancer (GLOBOCAN) in 2018, it was found that breast cancer ranks 2nd most of all cancers in the world with 2,088,849 new cases (11.6\%) accompanied by a death rate of 626,279 cases, while in Indonesia breast cancer ranks first with 58,256 new cases (19.8\%) and is also the leading cause of death in women with 22,692 cases $(12.75 \%)$ [3,4].

Breast cancer shows a heterogeneous disease from various entities with different morphological appearances and natural characteristics. Breast cancer in recent years, it has been proven that this diversity is caused by genetic changes [5]. 
Mucin 1 (MUC1) is a single pathway type I transmembrane glycoprotein expressed only on the apical surface of normal gland and ductal epithelial cells, is a potential target for cancer therapy, and as a 2nd rank cancer vaccine target among 75 other cancer antigens identified. assessed by the National Cancer Institute [6]. This rating is based on the aberrant glycosylation pattern of tumor-specific MUC1 in various forms of adenocarcinoma. Aberrant glycosylated MUC1 is overexpressed on the entire surface of tumor cells, in contrast to apical expression in normal cells [7]. Due to aberrant glycosylation, T-cell and B-cell epitopes on the peptide backbone are now accessible, forming the tumor-associated MUC1 antigen. Correspondingly, several clinical and preclinical studies have studied MUC1-mediated immunotherapy and cancer vaccination (reviewed in other organs, such as the lung) $[6,8]$.

In many tumor types, MUC1 expression correlates with cell aggressiveness, metastasis, poor response to therapy, and poor survival. Although MUC1 expression is limited to the apical surface of the most ductal epithelium, in metastatic disease, MUC1 is overexpressed and becomes localized throughout the cell [9]. MUC1 overexpression in breast cancer is the result of genetic alterations and transcriptional dysregulation. The MUC1 gene is located on chromosome 1q21, a frequently altered site in breast cancer cells [10].

Aberrant overexpression of MUC1 is associated with angiogenesis and chemoresistance in cancer. Thus MUC1 has a role in tumorigenesis, progression, and metastasis, serving as a prognostic factor [11]. In the United States, of the 1.4 million tumors diagnosed annually, approximately 0.9 million overexpress MUC1. Therefore, due to its ubiquity and role in cancer development, it has become an important target in the development of new therapies [12].

There are several studies regarding the correlation between MUC1 expression and the histological grade of breast carcinoma, which showed different results, including according to a study conducted by Manu et al., who found that there was no significant correlation between positive-expressed MUC1 and histological grade, although there was a tendency for MUC1 to be expressed positively, that cases with positive MUC1 expression were dominated by higher grades [6]. This contrasts with studies conducted by Kim et al., Syed et al., and Do et al., which showed that positive MUC1 expression was often associated with a lower grade of breast carcinoma [13-15].

Molecular subtypes in breast carcinoma are known to be associated with the prognosis of breast cancer patients, showing that luminal A has the best prognosis and basal-like or triple-negative has the worst prognosis [16]. According to Tan et al., heterogeneity of molecular subtypes in triple-negative breast cancer (TNBC) can influence cancer therapy and aggressiveness [17]. MUC1 expression is seen in all subtypes of breast cancer, including luminal, HER2+ (Human epidermal growth factor receptor 2), and basal, although in each of these cancer types, expression is highest in metastatic tumors [9].

This study aims to analyze the correlation between the immunohistochemical expression of Mucin 1 (MUC1) with histopathological grading and molecular subtypes in invasive breast carcinoma.

\section{Material and Methods}

Researchers examined 42 samples from invasive breast carcinoma at H. Adam Malik Hospital Medan which were diagnosed histopathologically by hematoxylin-eosin (HE) staining. This study is an analytical study with a cross-sectional approach to assessing the characteristics and immunohistochemical expression of MUC1, starting from January 2021 to October 2021. This research has been approved by the Ethics Committee for Health Research Implementation, Faculty of Medicine, Universitas Sumatera Utara with the number: 443/KEP/USU/2021. This study displays the characteristics of the sample, namely age, histopathological subtype, histopathological grading, molecular subtype, and Mucin 1 (MUC1) expression. The sample used is a sample that conforms to the inclusion and exclusion criteria. Inclusion criteria were all slides and paraffin blocks originating from post-mastectomy breast tissue diagnosed as histopathologically representative invasive breast carcinoma after staining with hematoxylin-eosin (HE) at H. Adam Malik Hospital Medan and having medical record data that included ER, PR, HER2, and Ki-67 
immunohistochemical examination data in patients with invasive breast carcinoma. Exclusion criteria were slides and paraffin blocks derived from core biopsy and lumpectomy of breast tissue diagnosed as histopathologically invasive breast carcinoma, slides and paraffin blocks that could not be re-evaluated or recut for examination with MUC1 immunohistochemical, and incomplete ER PR, HER2, and Ki-67 immunohistochemical examination data.

The immunohistochemical expression of MUC1 in this study was categorized into negative and positive. It is said to be negative if MUC1 expression does not appear brown or appears brown in the cell membrane and or of cytoplasm $\leq 10 \%$ of tumor cells. And it is said to be positive if MUC1 expression appears brown in the cell membrane and/or cytoplasm $>10 \%$ of tumor cells [18].

\section{Results}

This study involved 42 samples diagnosed histopathologically as invasive breast carcinoma that had met the inclusion criteria and exclusion criteria were collected until the number of samples was sufficient based on the minimum sample size. All samples were post-mastectomy preparations at H. Adam Malik Hospital Medan. Data on molecular subtypes (ER, PR, HER2, Ki-67) are secondary data from medical record data (pathology archive). In this study, the youngest patient with invasive breast carcinoma was 23 years old, while the oldest patient was 70 years old, with a mean age of 48.1 years, and a median age of 48.5 years. For the age group, the most found were in the age group 40-49 years and the age group 50-59 years, each with 15 cases (35.7\%), while the age group 20-29 years was the least, namely 1 case $(2.4 \%)$ (Table 1$)$.

Based on Table 1 below, the majority of the samples were subtypes of invasive breast carcinoma of no special type, 36 cases $(85.7 \%)$, followed by invasive lobular carcinoma with 4 cases $(9.5 \%)$, and mucinous carcinoma with 2 cases (4.8\%). Meanwhile, other subtypes were not found in this study. In histopathological grading, the most common was grade 2 with 18 cases (42.8\%), and the least encountered was grade 3 with 11 cases $(26.2 \%)$. The most common molecular subtypes were luminal B with 17 cases $(40.5 \%)$. Meanwhile, the least triple-negative molecular subtypes were found, namely 3 cases $(7.1 \%)$. The immunohistochemical expression of Mucin 1 (MUC1) which was displayed on the cell membrane and/or cytoplasm of tumor cells, was found to be negative in 11 cases $(26.2 \%)$. While positive expressions were found in 31 cases $(73.8 \%)$.

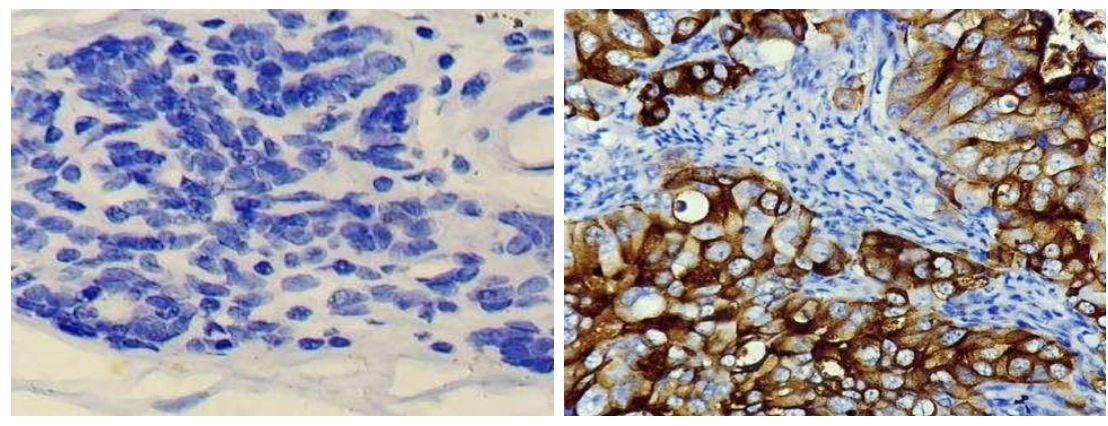

Figure 1. MUC1 immunohistochemical staining in invasive breast carcinoma. A. Negative MUC1 expression. B. Positive MUC1 expression. 
Table 1 Frequency distribution of characteristics of invasive breast carcinoma

\begin{tabular}{|c|c|c|}
\hline Sample characteristics & $\begin{array}{c}\text { Total } \\
\text { (n) }\end{array}$ & $\begin{array}{c}\text { Percentage } \\
(\%)\end{array}$ \\
\hline \multicolumn{3}{|l|}{ Age (years) } \\
\hline $20-29$ & 1 & 2.4 \\
\hline $30-39$ & 7 & 16.7 \\
\hline $40-49$ & 15 & 35.7 \\
\hline $50-59$ & 15 & 35.7 \\
\hline$\geq 60$ & 4 & 9.5 \\
\hline \multicolumn{3}{|l|}{ Histopathological subtypes } \\
\hline Invasive breast carcinoma of no special type & 36 & 85.7 \\
\hline Invasive lobular carcinoma & 4 & 9.5 \\
\hline Invasive tubular carcinoma & 0 & 0.0 \\
\hline Cribriform carcinoma & 0 & 0.0 \\
\hline Mucinous carcinoma & 2 & 4.8 \\
\hline Mucinous cystadenocarcinoma & 0 & 0.0 \\
\hline Invasive micropapillary carcinoma & 0 & 0.0 \\
\hline Carcinoma with apocrine differentiation & 0 & 0.0 \\
\hline Metaplastic carcinoma & 0 & 0.0 \\
\hline \multicolumn{3}{|l|}{ Histopathological grading } \\
\hline Grade 1 & 13 & 31.0 \\
\hline Grade 2 & 18 & 42.8 \\
\hline Grade 3 & 11 & 26.2 \\
\hline \multicolumn{3}{|l|}{ Molecular subtypes } \\
\hline Luminal A & 8 & 19.1 \\
\hline Luminal B & 17 & 40.5 \\
\hline HER2 enriched & 14 & 33.3 \\
\hline Triple-negative & 3 & 7.1 \\
\hline \multicolumn{3}{|c|}{ Mucin 1 (MUC1) immunohistochemical expression } \\
\hline Negative & 11 & 26.2 \\
\hline Positive & 31 & 73.8 \\
\hline
\end{tabular}

The frequency distribution of Mucin 1 (MUC1) immunohistochemical expression based on histopathological subtypes, it was found that from 42 samples showed invasive breast carcinoma of no special type the most were unexpressed/negative, namely 9 cases $(21.4 \%)$, followed by invasive lobular carcinoma with 2 cases $(4.8 \%)$, and in mucinous carcinoma, there were no case with negative expression $(0.0 \%)$. For positive expression Mucin 1 (MUC1) was most commonly found in the subtype of invasive breast carcinoma of no special type with 27 cases $(64.3 \%)$. While invasive lobular carcinoma and mucinous carcinoma each expressed positively with 2 cases $(4.8 \%)$. An assessment of the correlation between the immunohistochemical expression of Mucin 1 (MUC1) and histopathological subtypes was carried out using the Spearman's statistical test, and the results did not find a significant correlation between the two, where the p-value was 0.746 (p-value > 0.05) (Table 2). 
Table 2 Frequency distribution of Mucin 1 (MUC1) immunohistochemical expression based on histopathological subtypes in invasive breast carcinoma

\begin{tabular}{|c|c|c|c|c|c|c|c|c|c|}
\hline \multirow{3}{*}{$\begin{array}{l}\text { Mucin } 1 \text { (MUC1) } \\
\text { Immunohistochemical } \\
\text { Expression }\end{array}$} & \multicolumn{6}{|c|}{ Histopathological Subtypes } & \multicolumn{2}{|c|}{ Total } & \multirow[t]{3}{*}{ p-Value } \\
\hline & \multicolumn{2}{|c|}{$\begin{array}{l}\text { Invasive breast } \\
\text { carcinoma of no } \\
\text { special type }\end{array}$} & \multicolumn{2}{|c|}{$\begin{array}{l}\text { Invasive } \\
\text { lobular } \\
\text { carcinoma }\end{array}$} & \multicolumn{2}{|c|}{$\begin{array}{l}\text { Mucinous } \\
\text { carcinoma }\end{array}$} & & & \\
\hline & $\mathrm{n}$ & $\%$ & $\mathrm{n}$ & $\%$ & $\mathrm{n}$ & $\%$ & $\mathrm{n}$ & $\%$ & \\
\hline Negative & 9 & 21,4 & 2 & 4,8 & 0 & 0,0 & 11 & 26,2 & \\
\hline Positive & 27 & 64,3 & 2 & 4,8 & 2 & 4,8 & 31 & 73,8 & \\
\hline
\end{tabular}

Based on Table 3 below, from 42 samples, the positive expression of Mucin 1 (MUC1) immunohistochemical was found at most in grade 2 with 14 cases $(33.3 \%)$, followed by grade 3 with 10 cases (23.8\%), and grade 1 with 7 cases (16.7\%). As for the negative expression of Mucin 1 (MUC1), it was found that the most common was found in grade 1 with 6 cases $(14.3 \%)$, followed by grade 2 with 4 cases $(9.5 \%)$, and grade 3 with 1 case $(2.4 \%)$. \%). In this study, an assessment of the correlation between the immunohistochemical expression of Mucin 1 (MUC1) and histopathological grading in invasive breast carcinoma was carried out. From the results of Somers'd statistical test, it was found that the p-value was 0.027 ( $\mathrm{p}$-value $<0.05$ ). These results indicate that there is a significant unidirectional correlation between the immunohistochemical expression of Mucin 1 (MUC1) and histopathological grading in invasive breast carcinoma.

Table 3 Frequency distribution of Mucin 1 (MUC1) immunohistochemical expression based on histopathological grading in invasive breast carcinoma

\begin{tabular}{|c|c|c|c|c|c|c|c|c|c|}
\hline \multirow{2}{*}{$\begin{array}{l}\text { Mucin 1 (MUC1) } \\
\text { Immunohistochemical } \\
\text { Expression }\end{array}$} & \multicolumn{6}{|c|}{ Histopathological Grading } & \multicolumn{2}{|c|}{ Total } & \multirow[t]{2}{*}{ p-Value } \\
\hline & \multicolumn{2}{|c|}{ Grade 1} & \multicolumn{2}{|c|}{ Grade 2} & \multicolumn{2}{|c|}{ Grade 3} & $\mathrm{n}$ & $\%$ & \\
\hline Negative & 6 & 14,3 & 4 & 9,5 & 1 & 2,4 & 11 & 26,2 & \\
\hline
\end{tabular}

Table 4 Frequency distribution of Mucin 1 (MUC1) immunohistochemical expression based on molecular subtypes in invasive breast carcinoma

\begin{tabular}{|c|c|c|c|c|c|c|c|c|c|c|c|}
\hline \multirow{3}{*}{$\begin{array}{l}\text { Mucin 1 (MUC1) } \\
\text { Immunohistochemical } \\
\text { Expression }\end{array}$} & \multicolumn{8}{|c|}{ Molecular Subtypes } & \multicolumn{2}{|c|}{ Total } & \multirow[t]{3}{*}{ p-Value } \\
\hline & \multicolumn{2}{|c|}{ Luminal A } & \multicolumn{2}{|c|}{ Luminal B } & \multicolumn{2}{|c|}{$\begin{array}{c}\text { HER2 } \\
\text { enriched }\end{array}$} & \multicolumn{2}{|c|}{$\begin{array}{c}\text { Triple } \\
\text { negative }\end{array}$} & \multirow[b]{2}{*}{$\mathrm{n}$} & \multirow[b]{2}{*}{$\%$} & \\
\hline & $\mathrm{n}$ & $\%$ & $\mathrm{n}$ & $\%$ & $\mathrm{n}$ & $\%$ & $\mathrm{n}$ & $\%$ & & & \\
\hline Negative & 3 & 7,1 & 7 & 16,7 & 1 & 2,4 & 0 & 0,0 & 11 & 26,2 & \\
\hline Positive & 5 & 11,9 & 10 & 23,8 & 13 & 30,9 & 3 & 7,1 & 31 & 73,8 & \\
\hline
\end{tabular}


The results of the study on the immunohistochemical expression of Mucin 1 (MUC1) with molecular subtypes from Table 4 show that from 42 samples, the most positive expression was found in the HER2 enriched molecular subtype with 13 cases (30.9\%), followed by luminal B with 10 cases $(23,8 \%)$, luminal A with 5 cases $(11.9 \%)$, and triple-negative with 3 cases $(7.1 \%)$. For immunohistochemical expression of negative Mucin 1 (MUC1), the most found in molecular subtype luminal B with 7 cases (16.7\%), followed by luminal A with 3 cases (7.1\%), HER2 enriched with 1 case $(2.4 \%)$, while the triple-negative was not found $(0.0 \%)$. Based on the Somers 'd statistical test, the p-value was 0.010 (p-value > 0.05), this result showed a significant unidirectional correlation between the immunohistochemical expression of Mucin 1 (MUC1) and the molecular subtypes in invasive breast carcinoma.

\section{Discussion}

In this study, from 42 samples of invasive breast carcinoma, the most common age group was 40-49 years old and 50-59 years old (35.7 each), while the age group 20-29 years was the least ( $2.4 \%)$. The results of this study are in line with research from Fandini, with the 40-49 year age group being the most common (37.7\%), and the 20-29 year age group is the least (1.8\%) [19]. This is in accordance with the literature statement, which states that the age of over 30 years will show an increased risk of developing invasive breast carcinoma compared to women under the age of 30 years, although this carcinoma can occur at any age [2].

Breast cancer is a heterogeneous group of neoplasms containing various cancers, characterized by very different biological characteristics, clinical course, and prognoses. Most breast cancers are invasive, and there are several histopathological subtypes of invasive breast cancer. The most common histopathological subtype of breast cancer is invasive breast carcinoma of no special type (NST). No special type is a group of cancers that do not show certain specific features of other histological types of breast cancer. Approximately 8 out of 10 invasive breast cancers are invasive breast carcinoma NST [20,21]. This statement is in accordance with the results of the research conducted, namely the histopathological subtype of invasive breast carcinoma NST as the most common subtype (85.7\%).

Breast cancer is the most studied malignancy. Despite improvements in the treatment of this cancer, breast cancer is still a major cause of mortality and morbidity among women with cancer. This can be caused by many factors, and one of them can be caused by the occurrence of resistance to treatment and inadequacy of treatment, or in cancer where distant metastases have occurred so that it is in an advanced stage. Although efforts are underway to identify the factors responsible for the aggressiveness of these cancers, the molecular events underlying the development of these cancers remain unclear. Mucins have emerged as important molecules in the development and metastasis of breast cancer. An expression change, glycosylation, is currently being actively studied to better understand its role in breast cancer pathogenesis. Current and emerging evidence suggests that mucins are differentially expressed during the development and metastasis of breast cancer, and thus, could be very useful both in early detection and/or predicting the prognosis of breast cancer [22].

This study was conducted to find the correlation between the immunohistochemical expression of MUC1 which is a mucin family with invasive breast carcinoma, in this case, related to histopathological grading and molecular subtypes. Previously, many studies were carried out related to MUC1 in cancer, one of which was breast cancer as previously mentioned above, and this continues to be developed in the form of research, to find the best management in order to get a good clinical outcome. MUC1 expression in cancer is changed from being confined to the apical surface to being expressed throughout the cell surface, and this expression may play a role in the occurrence of metastasis in cancer. From the results of this study, it was found that as many as $73.8 \%$ of patients with invasive breast carcinoma expressed positive MUC1. With the finding of $73.8 \%$ MUC1 immunohistochemical expression, it is possible for these patients to have a poor prognosis in the future. 
In this study, statistical tests were conducted to examine the correlation between MUC1 immunohistochemical expression and histopathological grading in invasive breast carcinoma. The results obtained showed a significant unidirectional relationship ( $\mathrm{p}$-value $=0.027$ ). These results are in line with the research conducted by Rakha et al., with the results obtained a significant correlation between MUC1 immunohistochemical expression and histopathological grading in invasive breast carcinoma ( $p$-value $<0.001$ ) [23]. In several other studies, results were not in line with this study, namely the research conducted by Manu et al., Do et al., and Patel et al., where the results showed that there was no significant correlation (with pvalues of $0.889 ; 0.077$; and 0.153$)[6,15,24]$.

Altered mucin expression has been reported to be associated with cancer progression, which in turn affects cell growth, differentiation, transformation, adhesion, invasion, and immune surveillance. Mucin 1 (MUC1) is the most studied in breast cancer. Several research groups have revealed that mucin has an important role in pathological conditions and has great potential as a prognostic marker, and as a therapeutic target in breast cancer. MUC1 is one of the best mucin families concerning its role in signal transduction in breast cancer cells. Overexpression of MUC1 in breast cancer cells has been shown to block cell death in response to oxidative stress, DNA damage, hypoxia, induce anchorage-independent growth, and tumorigenicity [22]. The finding of a significant unidirectional correlation between MUC1 immunohistochemical expression and histopathological grading indicates that MUC1 expression can detect early breast cancer prognosis more accurately, where the higher the histopathological grading, the more MUC1 will be expressed, and vice versa. So, the more expressed MUC1 the worse the prognosis for patients with invasive breast carcinoma, and vice versa.

The results of this study also looked for the correlation between MUC1 expression and molecular subtypes using the Somers'd test, and the results showed that there was a significant unidirectional correlation between MUC1 immunohistochemical expression and molecular subtypes (luminal A, luminal B, HER2 enriched, triple-negative) in invasive breast carcinoma ( $\mathrm{p}$-value $=0.010)$. This indicates that the worse the molecular subtype, the more MUC1 is expressed, and vice versa. The results of this study are not in line with the research conducted by Elseed et al., which showed that there was no significant correlation ( $\mathrm{p}$-value $=0.5$ ). Meanwhile, according to a study conducted by Manu et al., there was a significant correlation between MUC1 expression with HER2 enriched and triple-negative molecular subtypes ( $\mathrm{p}$-value $=0.01$ and $<0.05$ ), but there was no significant correlation with luminal A and luminal B (p-value $=0.828$ and 0.787 ) $[6,25]$.

Women with tumors showing ER, PR, and HER2 negative (triple-negative) did not receive additional maintenance therapy after adjuvant chemotherapy. This is an important consideration because triple-negative breast cancer is associated with a more aggressive phenotype and has a higher risk of distant metastases and decreased survival. Given the high risk of recurrence associated with early-stage triple-negative breast cancer, it would be advantageous to adopt preventive strategies, including immunotherapy to reduce the risk of recurrence. The success of an immune-based approach to breast cancer therapy depends on the identification of a broadly expressed tumor antigen that can be targeted at breast cancer cells, and MUC1 is that target, expressed in many adenocarcinomas that can induce a specific immune response [26].

As mentioned above, breast cancer still shows high mortality and morbidity today. This could be due to the occurrence of treatment resistance. And one of the causes could be due to aberrant MUC1 expression due to overexpression in breast cancer. The hydrophilic extracellular VNTR area of MUC1 and its high glycosylation can prevent hydrophobic chemotherapy drugs from crossing the membrane and into the cell. MUC1 expression in breast cancer cells with circulating metastases correlates with a lack of treatment effectiveness. In breast cancer patients with no positive MUC1 found, it will show tumor cells respond better to chemotherapy [27,28]. By obtaining a significant unidirectional correlation between MUC1 immunohistochemical expression and molecular subtypes in this study, it can be estimated that this invasive breast carcinoma patient may undergo treatment resistance. Understanding the resistance mechanisms involving mucins will contribute to the development of targeted therapies, and can help in prevention because 
of their potential as tumor biomarkers to direct therapeutic options towards potentially more successful therapies, and can avoid more aggressive alternative therapies, which will fail [29].

\section{Conclusion}

From the results of this study, it was found that the immunohistochemical expression of Mucin 1 (MUC1) was positive in $73.8 \%$ of invasive breast carcinomas, and there was a significant correlation between the immunohistochemical expression of Mucin 1 (MUC1) with histopathological grading and molecular subtypes of invasive breast carcinoma. The results of this study indicate that MUC1 can be used as one of the predictive factors for an accurate prognosis of invasive breast carcinoma, as well as one of the therapies in the form of anti-MUC1, especially in triple-negative molecular subtypes that can be used, either in the form of targeted therapy or immunotherapy to prevent the occurrence of treatment resistance and recurrence in invasive breast carcinoma.

\section{Acknowledgments}

We acknowledgments to all staff and residents of the Department of Pathology Anatomics, Universitas Sumatera Utara / H. Adam Malik Hospital Medan for all your support and guidance.

\section{REFERENCES}

1. Bray F, Ferlay J, Soerjomataram I, Siegel RL, Torre LA, Jemal A. Global cancer statistics 2018: GLOBOCAN estimates of incidence and mortality worldwide for 36 cancers in 185 countries. CA Cancer J Clin. 2018; 68: pp. 394-424.

2. Colditz G, Chia KS, Wilson R, Britton P, Morrow M, Rutgers E, et al. Invasive breast carcinoma: introduction and general features. In: Lakhani SR, Ellis IO, Schnitt SJ, Tan PH, Vijver MJ, editors. WHO classification of tumours of the breast. $4^{\text {th }}$ ed. Lyon: IARC. 2012; pp. 14-23.

3. WHO. Breast cancer. IARC. 2018. Available from: http://gco.iarc.fr/today/data/factsheets/cancers/20-Breast-fact-sheet.pdf [Accessed April 9 ${ }^{\text {th }}$ 2019].

4. WHO. Indonesia. IARC. 2018. Available from: http://gco.iarc.fr/today/data/factsheets/populations/360-indonesia-factsheets.pdf [Accessed April 9 ${ }^{\text {th }} 2019$ ].

5. Gannon LM, Cotter MB, Quinn CM. The classification invasive carcinoma of the breast. Expert Rev Anticancer Ther. 2013; 13(8): pp. 941-54.

6. Manu EA, Bedu-Addo K, Titiloye NA, Ameh-Mensah C, Opoku F, Duduyemi BM. Expression of tumour-associated MUC1 is a poor prognostic marker in breast cancer in Kumasi, Ghana. J. Onco. 2020; [7 p.].

7. Sousa AM, Grandgenett PM, David L, Almeida R, Hollingsworth MA, Santos-Silva F. Reflection on MUC1 glycoprotein: the hidden potential of isoforms in carcinogenesis. APMIS. 2016; 72(124): pp. 913-24.

8. Hossain MdK and Wall KA. Immunological evaluation of recent MUC1 glycopeptide cancer vaccines. Vaccines. 2016; 4(25): [13 p.].

9. Horm TM and Schroeder JA. MUC1 and metastatic cancer expression, function and therapeutic targeting. Cell Adh \& Migr. 2013; 7(2): pp. 187-98.

10. Kufe DW. MUC1-C oncoprotein as a target in breast cancer: activation of signaling pathways and therapeutic approaches. Oncogen. 2013; 32: pp. 1073-81.

11. Goode G, Gunda V, Chaika NV, Purohit V, Yu F, Singh PK. MUC1 facilitates metabolic reprogramming in triple-negative breast cancer. PLoS One. 2017; [15 p.].

12. Pillai K, Pourgholami MH, Chua TC, Morris DL. MUC1 as a potential target in anticancer therapies. Am J Clin Oncol. 2015; 38: pp. 108-18.

13. Kim D, Jung W-H, Koo JS. Expression of MUC1, MUC2, MUC5AC and MUC5B in mucinous lesions of the breast. Pathobiology. 2012; 79(3); pp. 144-53.

14. Syed BM, Green AR, Paish EC, Soria D, Garibaldi J, Morgan L, et al. Biology of primary breast cancer in older women treated by surgery: with correlation with long-term clinical outcome and comparison with their younger counterparts. BJC. $2013 ; 108(5)$ : pp. 1042-51 
15. Do SI, Kim K, Kim DH, Chae SW, Park YL, Park CH, et al. Associations between the expression of mucins (MUC1, MUC2, MUC5AC, and MUC6) and clinicopathologic parameters of human breast ductal carcinomas. J Breast Cancer. 2013; 16(2): pp. 152-8.

16. Lefebvre C, Bachelot T, Filleron T, Pedrero M, Campone M, Soria J, et al. Mutational profile of metastatic breast cancers: a retrospective analysis. PLosMed. 2016; [18 p.].

17. Tan B, Kanoko M, Tan G, Bachtiar A, Munir D. Dimensional analysis of CD44 high CD24 low and Ki67 in triple negative breast cancer. Maced J Med Sci. 2019; 7(4): pp. 526-8.

18. Van der Vegt B, de Roos MAJ, Peterse JL, Patriarca C, Hilkens J, de Bock GH, et al. The expression pattern of MUC1 (EMA) is related to tumour characteristic and clinical outcome of invasive ductal breast carcinoma. Histopathology. 2007; 51: pp. 322-35.

19. Fandini I. Karakteristik penderita kanker payudara di RSUP H. Adam Malik tahun 2016. Medan: Universitas Sumatera Utara. 2017. Available from: http://repositori.usu.ac.id/handle/123456789/4539 [Accessed June $25^{\text {th }} 2019$ ].

20. Harbeck N and Gnant M. Breast cancer. Lancer. 2017; 389: pp. 1134-50.

21. American Cancer Society. About breast cancer. Available https://www.cancer.org/content/dam/CRC/PDF/Public/8577.00.pdf [Accessed September 28 ${ }^{\text {th }} 2021$ ].

22. Mukhopadhyay P, Chakraborty S, Ponnusamy MP, Lakshmanan I, Jain M, Batra SK. Mucins in the pathogenesis of breast cancer: implications in diagnosis, prognosis and therapy. Biochim Biophys Acta. 2011; 1815(2): pp. 224-40.

23. Rakha EA, Boyce RWG, El-Rehim DA, Kurien T, Green AR, Paish EC, el al. Expression of mucins (MUC1, MUC2, MUC3, MUC4, MUC5AC and MUC6) and their prognostic significance in human breast cancer. Modern pathology. 2005; 18: pp. 1295-1304.

24. Patel DS, Khandeparkar SGS, Joshi AR, Kulkarni MM, Dhande B, Lengare P. Immunohistochemical study of MUC1, MUC2 and MUC5AC expression in primary breast carcinoma. J Clin and Diag Res. 2017; 11(4): pp. 30-4.

25. Elseed SMH, Abdelaziz MS, Salih NS, Elnaeem MM, El-fatih M, Mudawi AI. Mucin 1 (MUC1) over expression and intracellular localization pattern in invasive ductal breast cancer using immunohistochemistry. American Journal of Research Communication. 2015; 3(7): [8.p.].

26. Siroy A, Abdul-Karim FW, Miedler J, Fong N, Fu P, Gilmore H, et al. MUC1 is expressed at high frequency in early-stage basal-like triple-negative breast cancer. Human Pathology. 2013; 44: pp. 2159-66.

27. Apostolopoulos V, Stojanovska L, Gargosky SE. MUC1 (CD227): a multi-tasked molecule: review. Cellular and Molecular Life Sciences. Springer. 2015; [26 p.].

28. Cheng JP, Yan Y, Wang XY, Lu YL, Yuan YH, Jia J, Ren J. MUC1-positive circulating tumor cells and MUC1 protein predict chemotherapeutic efficacy in the treatment of metastatic breast cancer. Chin J Cancer. 2011; 30: pp. 54-61.

29. Jonckheere N, Skrypek N, Seuningen I. Mucins and tumor resistance to chemotherapeutic drugs. Biochimica et Biophysica Acta, Elsevier. 2014: [10 p.]. 